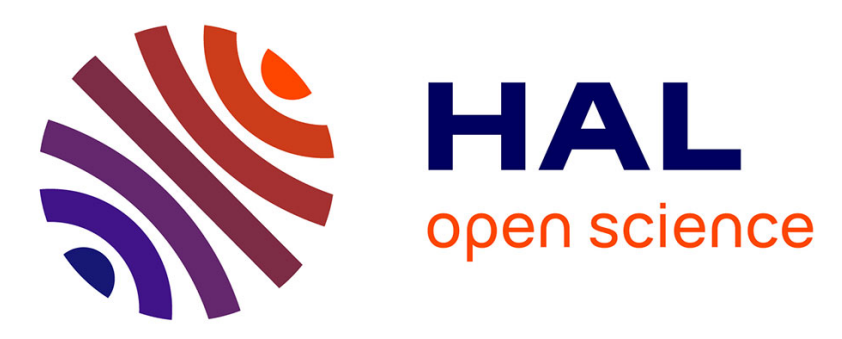

\title{
Terahertz Pulses Emitters with Full Electrical Control on Polarization for THz-TDS
}

Kenneth Maussang, J. Palomo, Jean-Michel Manceau, Raffaele Colombelli, Isabelle Sagnes, L.H. Li, Edmund H. Linfield, A. Giles Davies, J. Mangeney, J. Tignon, et al.

\section{To cite this version:}

Kenneth Maussang, J. Palomo, Jean-Michel Manceau, Raffaele Colombelli, Isabelle Sagnes, et al.. Terahertz Pulses Emitters with Full Electrical Control on Polarization for THz-TDS. 2018 43rd International Conference on Infrared, Millimeter, and Terahertz Waves (IRMMW-THz 2018), Sep 2018, Nagoya, France. pp.1-2. hal-02127991

\section{HAL Id: hal-02127991 https://hal.umontpellier.fr/hal-02127991}

Submitted on 23 May 2019

HAL is a multi-disciplinary open access archive for the deposit and dissemination of scientific research documents, whether they are published or not. The documents may come from teaching and research institutions in France or abroad, or from public or private research centers.
L'archive ouverte pluridisciplinaire HAL, est destinée au dépôt et à la diffusion de documents scientifiques de niveau recherche, publiés ou non, émanant des établissements d'enseignement et de recherche français ou étrangers, des laboratoires publics ou privés. 


\title{
Photoconductive switches for polarization resolved THz time-domain spectroscopy
}

\author{
K. Maussang ${ }^{1}$, J. Palomo ${ }^{2}$, J.-M. Manceau ${ }^{3}$, R. Colombelli ${ }^{3}$, I. Sagnes ${ }^{3}$, L.H. Li ${ }^{4}$, E.H. Linfield ${ }^{4}$, \\ A.G. Davies ${ }^{4}$, J. Mangeney ${ }^{2}$, J. Tignon ${ }^{2}$ and S. S. Dhillon ${ }^{2}$ \\ ${ }^{1}$ Institut d'Electronique et des Systèmes, Université de Montpellier, \\ 860 rue de Saint-Priest, 34095 Montpellier Cedex 5, CNRS, Montpellier, France \\ ${ }^{2}$ Laboratoire Pierre Aigrain, Ecole Normale Supérieure-PSL Research University, CNRS, Université Pierre et \\ Marie Curie-Sorbonne Universités, Université Denis Diderot-Sorbonne Paris Cité, \\ 24 rue Lhomond, 75231 Paris Cedex 05, France \\ ${ }^{3}$ Centre de Nanosciences et de Nanotechnologies, CNRS, Univ. Paris-Sud, Université Paris-Saclay, \\ C2N-Orsay, 91405 Orsay Cedex, France \\ ${ }^{4}$ School of Electronic and Electrical Engineering, University of Leeds, Leeds LS9 2JT, United-Kingdom
}

\begin{abstract}
Photoconductive switches are widely used for emission and/or detection of terahertz pulses. The emitted polarization is fixed by the design of the electrodes. In this work, innovative designs of photoconductive switches are proposed providing full electrical control on the direction of polarization of the emitted field and its detection. These designs are based on monolithic on-chip solution with an interdigitated scalable geometry. It allows fast polarization modulation ability without the need of external mechanical components, and polarimetry measurements with a large area receiver. It opens the fields of precision terahertz polarimetry.
\end{abstract}

T NTERDigitated photoconductive (iPC) switches are powerful and convenient devices for time-resolved spectroscopy, with the ability to operate both as sources and detectors of terahertz $(\mathrm{THz})$ frequency pulses. Such iPC switches have been demonstrated with large areas for powerful emission, and may provide high-resolution performances [1]. Polarimetry and birefringence measurements in the $\mathrm{THz}$ range are of huge interest, especially in the field of material sciences. Most of polarization measurements in $\mathrm{THz}$ domain are performed by the use of mechanically controlled elements, which limits inherently the swiftness and the precision of the measurement. While standard iPC switches emit linearly polarized $\mathrm{THz}$ radiation, an innovative concept of a $\mathrm{THz}$ pulse emitter with a full electrical control over the emitted polarization is proposed and demonstrated over a $100 \mathrm{GHz}-4$ $\mathrm{THz}$ spectral window. Its design is scalable for large area devices, and enables decorrelation between the frequency bandwidth of operation and the size of the active area. It is based on an intermixed geometry of small interdigitated active area, as illustrated in figure 1. It consists in a subwavelength spatial alternation of small iPC switches with orthogonal direction of emitted polarization. In the far-field regime, this spatial distribution is no longer distinguishable, providing a homogeneous beam, resulting from the linear superposition of each type of polarization. From Malus' law, the direction of polarization is determined by the relative amplitude of the field of each type of emitter. In the linear regime, this amplitude is proportional to the electrical bias field applied by the iPC switches electrodes, and consequently the applied voltage. It results in a $\mathrm{THz}$ pulse emitter which polarization is fully electrically controlled without the need of additional mechanical element such as a wire-grid polarizer. This design might be adapted for detection scheme with iPC switches, providing the ability to measure simultaneously two orthogonal polarizations of the $\mathrm{THz}$ pulse, with a large area detector. The designs of iPC switches in this work are fully scalable, providing large area devices, both in emission and detection. Spatial polarization distortion has been evaluated numerically.
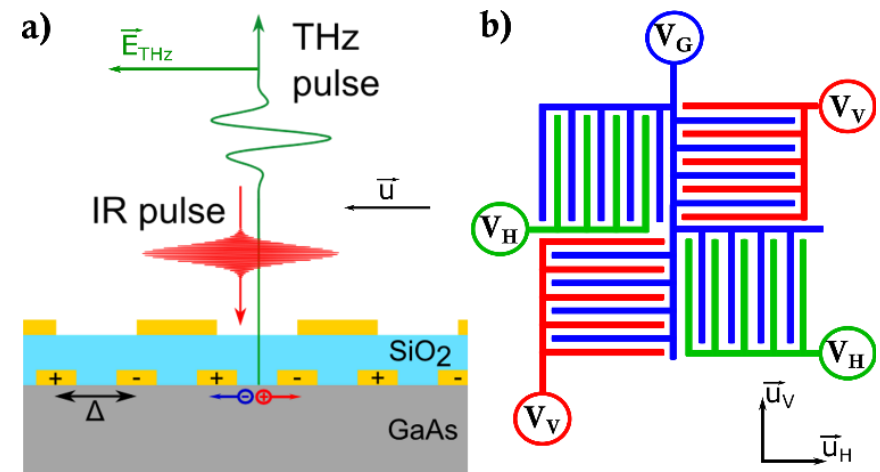

Fig. 1. a) Cut view of an interdigitated photoconductive switch. Interdigitated gold electrodes on top of the GaAs layer consist of $4 \mu \mathrm{m}$ wide electrodes, equally spaced by a distance $\Delta=4 \mu \mathrm{m}$. A second metallic layer is composed of metallic fingers covering gaps with a periodicity double that of the first, isolated from the first metallic layer by a $300 \mathrm{~nm}$ thick layer of $\mathrm{SiO}_{2}$. A femtosecond excitation pulse is focused on the front face of the photoconductive switch generating carriers in the GaAs layer (electrons in blue and holes in red). The $\mathrm{THz}$ pulse is emitted from the surface. b) Top view of the intermixed geometry principle (only the first metallic layer is represented). It consists in a pattern made out of two orthogonal small interdigitated photoconductive switches. The pairs of digits share a common ground potential $\mathrm{V}_{\mathrm{G}}$, but might be polarized independently with two different electrical potential $\mathrm{V}_{\mathrm{H}}$ and $\mathrm{V}_{\mathrm{V}}$, resulting is respectively horizontal and vertical polarization.

From its fast electrical modulation ability, these iPC switches would permit fast and high-precision polarization measurements. Such precision $\mathrm{THz}$ polarimetry opens the field of material sciences studies such as polymers or anisotropic materials like wood. Finally, reflectivity measurements for different polarizations permit to retrieve the dielectric constant of a material from Fresnel's coefficient without the need for a reference [2].

\section{REFERENCES}

[1]. K. Maussang et al., "Monolithic Echo-less Photoconductive Switches as a High-Resolution Detector for Terahertz Time-domain Spectroscopy," Appl. Phys. Lett., vol. 110, 141102, 2017.

[2]. W. Lai et al., "A novel method to measure dielectric properties of materials in terahertz spectroscopy," Optik, vol. 124, 4085-4088, 2013. 